\title{
PHYSICAL FACTORS IN TYRE TRACTION
}

\section{K C Ludema}

Department of Mechanical Engineering, University of Michigan, Ann Arbor, Michigan, USA

The ability of an automobile tyre to 'grip' the road and avoid skidding is ultimately dependent upon the events in the tyre-road interface. This seems obvious, and yet some 70 years after pneumatic rubber tyres were first put to use we are not able to explain fully the frictional processes between the tyre and the road. Over 650 papers have been published prior to 1970 on some aspect of tyre behaviour (Ludema and Gujrati 1973). The majority of these have been concerned with the development of tyres by empirical methods, and with the tyre as a structural element that influences vehicle dynamics. It will be several years before equations are developed from first principles to aid in tyre tread design, road texture specification and materials selection. This paper will describe some of the variables to be aware of in tyre performance, and it provides a "state of the art' review of the friction processes involved.

Considerable uncertainty exists concerning the importance of tyre-road traction (or friction). Intuitively most users of vehicles would endorse high friction on the simple ground that it would increase safety. However, there has not yet been a complete study of driver-vehicle-environment system effectiveness to determine how high friction should be. Nor has there been an effort to determine alternatives to high friction. The end result of an incompletely effective system is collision. In industrialized nations one person in 5000 is killed each year in automobile 'accidents' and many others are maimed. It is known that in many accidents a high tyre-road friction would not have reduced the severity of the consequences. In many other instances high friction might have been useful, but only if the driver had been trained to handle his vehicle during extreme manoeuvring. Few operators are experienced in vehicle manoeuvres involving accelerations in excess of $0.3 \mathrm{~g}$.

A major activity in the United States at this time is the specification of product quality. The tyre industry has been under some pressure to specify the limits and abilities of their product in a manner that is meaningful to the general public. Unfortunately the tyre is a very complex structure and its role in the dynamics of vehicle handling is only slowly becoming understood. However, the more difficult problem is to describe properly true behaviour over the wide range of operating conditions of tyres. Some of these conditions will be discussed in connection with the results of the skid resistance tests described in the following paragraphs. Many devices have been built to measure the traction ability of a tyre. Usually, when the results of such test programmes are used to try to predict the behaviour of a vehicle equipped with the tyres tested, poor correlation is found. Apparently the discrepancy lies in the fact that with full scale vehicles, many more dynamic quantities are at work than in the test devices. The tyre is a deformable body and it bears a fraction of the weight of the vehicle that varies with time. Roads are never perfectly smooth, vehicles never proceed in a straight line, and with every pitch, roll and bounce of the vehicle a great number of dynamic states is imposed upon the tyre. As a result, the tyre could appear to produce a higher or a lower friction in a full scale vehicle test than in a carefully controlled test with a single tyre. An extreme analogy of the tyre is a pogo stick. To ask what is the average friction between the tyre and the road is somewhat equivalent to inquiring as to the average friction between the pogo stick and the pavement.

\section{The skid resistance measurement of tyres}

There is a great interest in data on the tractive coefficient for a particular tyre on a particular road. However, complete data are difficult and expensive to obtain. Measuring a single value is not a useful exercise because tyre traction varies in an unpredictable manner with speed, amount of water on the road and many other factors. Illustrative data are given here for two conditions of tyre behaviour. 
First, it is known that average locked wheel sliding forces vary with speed on virtually all but the roughest road surface textures. Typical data are shown in figure 1 (Holmes and Stone 1968) for tractive coeffcient (equivalent to friction coefficient) over a range of initial vehicle speeds. Data are shown for 'dry' and for wet roads for two states of wear of the tyre. Note the great difference in performance of 'dry' versus wet roads. Also note that the amount of tread determines the speed at which very little tractive force is available for vehicle control. The condition where very low tractive coefficient occurs is often referred to as hydroplaning (or aquaplaning).

The braking force of a tyre varies with its speed of rotation relative to the speed of rotation of a freely rotating tyre. For example, if a vehicle is proceeding at 60 mile $\mathrm{h}^{-1}$ and if one tyre on that vehicle is rotating at a speed consistent with a vehicle speed of 54 mile $^{-1}$ that tyre is said to be 'slipping' $10 \%$ or $S=0 \cdot 10$. During normal braking of a vehicle, most of the tyres are slipping to some extent, of the order of $5 \%$ or less. In fact, a braking force cannot be generated without such slip. A freely rolling tyre has $S=0$ and a locked wheel has $S=1$. The tractive coefficient in braking over this full slip range is shown in figure 2 (Byrdsong 1968) for wet and dry concrete at 30 mile $^{-1}$.

It is apparent from figures 1 and 2 that water is an effective lubricant between the tyre and the road surface. However, the difference between dry road friction and wet road friction is not marked by the complete absence versus the presence of water. Rather it is a matter of the amount of water present. A surface that appears dry simply has less water than is visible. If we assume that the thickness of water films on 'dry' surfaces is on the order of $100 \AA$ $(=1 / 50$ the wavelength of green light), we may proceed to calculate the coefficient of friction between the tyre and the road if the only resistance to sliding

Figure 1 Tractive coefficient (tractive force/vehicle weight) over a range of vehicle speeds for all tyres on dry roads, and for good and bald tyres on wet roads

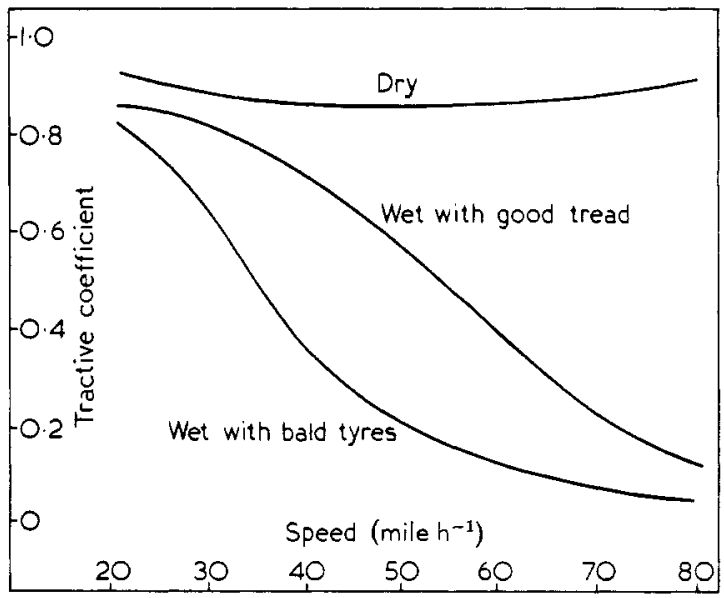

12

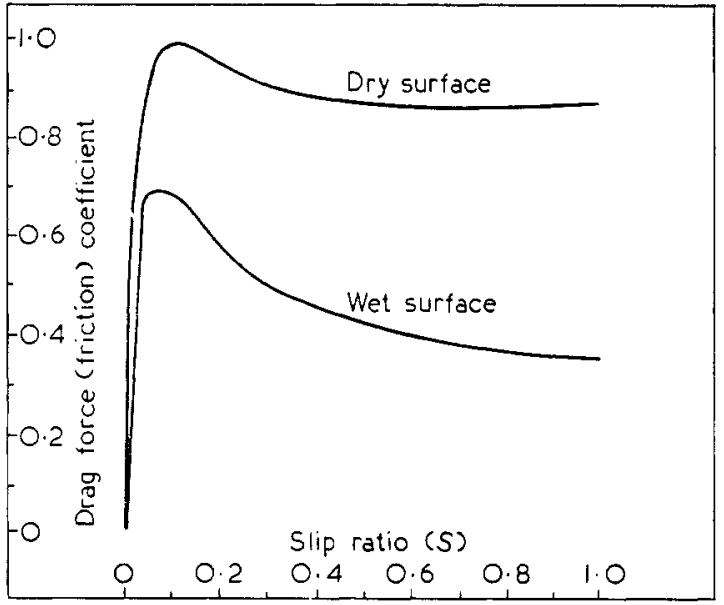

Figure 2 Drag force coefficient plotted against slip ratio on both wet and dry surfaces

is viscous drag in the fiuid film. Using an equation due to Clerk Maxwell we may calculate the tangential force $F$ due to viscous drag by the equation, $F=\eta A v / h$ where $\eta$ is the viscosity of the fluid, $A$ is the wetted area, $v$ is the sliding velocity and $h$ is the fluid film thickness. For a typical American tyre bearing a load of $460 \mathrm{~kg}$ the footprint of the tyre has a cross sectional area of about $1.4 \times 10^{-2} \mathrm{~m}^{2}$. The tyre is supported by a rough road surface texture, and it is safe to assume that intimate contact between the tyre and road exists over about $1 / 10$ of the footprint area. We may also reasonably assume that in the region of intimate contact the water film has been reduced to $10 \AA$ thickness. Further for convenience, we can estimate the sliding speed of the tyre to be the vehicle speed $v$, multiplied by $S$. A reasonable value of $S$ to achieve high friction is 0.1 as seen in figure 2 so that for $v=40 \mathrm{mile} \mathrm{h}^{-1}$ and $S=0 \cdot 1, v S=$ $1.60 \mathrm{~m} \mathrm{~s}^{-1}$. The viscous drag force then is $400 \mathrm{~kg}$, which with a normal load of $460 \mathrm{~kg}$ yields a coefficient of friction of 0.86 and this is a reasonable value for tyres on 'dry' roads.

Obviously there is considerable latitude in estimating water film thickness on road surfaces since such measurements have not yet been made. However, one can see that viscous drag can play an important role in the production of tractive forces between tyres and roads. Given the above calculation it is not necessary to define the difference between 'dry' roads and wet roads in terms other than the thickness of water film present. This concept aids considerably in deriving models of the friction behaviour between the tyre and the road. In most literature one sees suggestions that high friction between the tyre and the road may be achieved by squeezing the water film out completely. Again, one often sees references to the road surface asperities 'reaching through' the water film to 'touch' the tyre and thereby achieving local dry friction. Thus 
several authors have been tempted to explain traction of tyres on wet roads in terms of that fraction of the length of footprint from which all water has been expelled and dry friction exists. Apart from the conceptual difficulty of admitting the complete disappearance of absorbed films from contacting surfaces, there is the problem of the mathematical discontinuity required to explain the above state of affairs.

It has been suggested above that viscous drag is a prominent source of friction force between tyres and roads, and that 'dry' friction does not exist in this case. To place these comments in context it is necessary to discuss alternative mechanisms of friction.

\section{Mechanism of rubber friction}

For rubbing systems in general, there are several factors that contribute to sliding resistance. The friction of tyre rubber on practical surfaces can be divided into at least four components, or constituent parts. The naming of these four components is arbitrary to some extent, and they are here separated in terms of friction force (where $F_{10 t}$ is the total friction force; $F_{\text {ad }}$ the adhesive component; $F_{\mathrm{det}}$ the deformation component: $F_{\mathrm{vis}}$ the viscous component; and $F_{\mathrm{tear}}$ the tearing component) rather than the coefficient of friction:

$$
F_{\mathrm{tot}}=F_{\mathrm{ad}}+F_{\mathrm{def}}+F_{\mathrm{vis}}+F_{\mathrm{tear}} \text {. }
$$

Each component is defined above. Each component is distinctive as shown by simple experiments. Each component varies in a different way, with variation in contact stress, sliding speed, temperature, tyre material, road surface profile, and so on. In general these different responses to sliding speed, and so on, suggest very useful means of separating the several components by well chosen experiments. The several components of friction will first be described individually, for clarity. In later paragraphs, the likely balance of the components will be discussed in relation to a real tyre.

\section{The adhesive component of friction}

The existence of the adhesive, or dry, component of rubber friction is verified, and values are measured, by sliding a rubber specimen on a carefully cleaned, smooth surface, such as glass, in a vacuum. Such experiments show that a thin smooth film of rubber is often deposited, and remains attached to the mating surface. The sliding retardation force varies considerably with contact pressure, sliding speed and temperature, and is consistent with the view that the value of $F_{\text {ad }}$ is dependent on the viscoelastic mechanical properties of the polymer.

The clearest manifestation of a viscoelastic effect in friction is due to Grosch (1963). Figure 3a is taken from the work of Grosch and it shows friction data for an acrylonitrile-butadiene rubber sliding on glass at various temperatures over a speed range. Figure $3 \mathrm{~b}$ is a 'master curve' made by transforming the data for each temperature in figure $3 a$ by a different amount $\left(a_{T}\right)$ according to the method of the WLF (William, Landell and Ferry) transform. The conclusions of Grosch have been verified by several authors, and there is general agreement that successful application of the WLF rate-temperature equivalence concept clearly establishes the viscoelastic nature of the adhesive component of friction.

When a sliding body leaves a thin film or track, a friction test may merely be a shear test. If this is the

Figure 3a The coefficient of friction $(\mu)$ for acrylonitrilebutadiene rubber over a range of speeds, and at various temperatures. $b$ The data in a transformed by the WLF method to a reference temperature of $25^{\circ} \mathrm{C}$

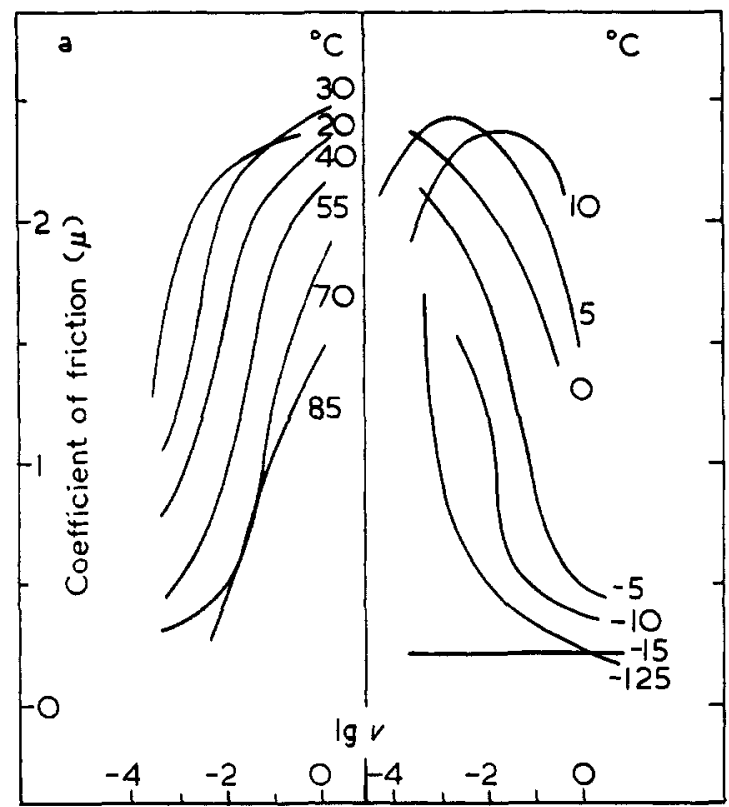

b

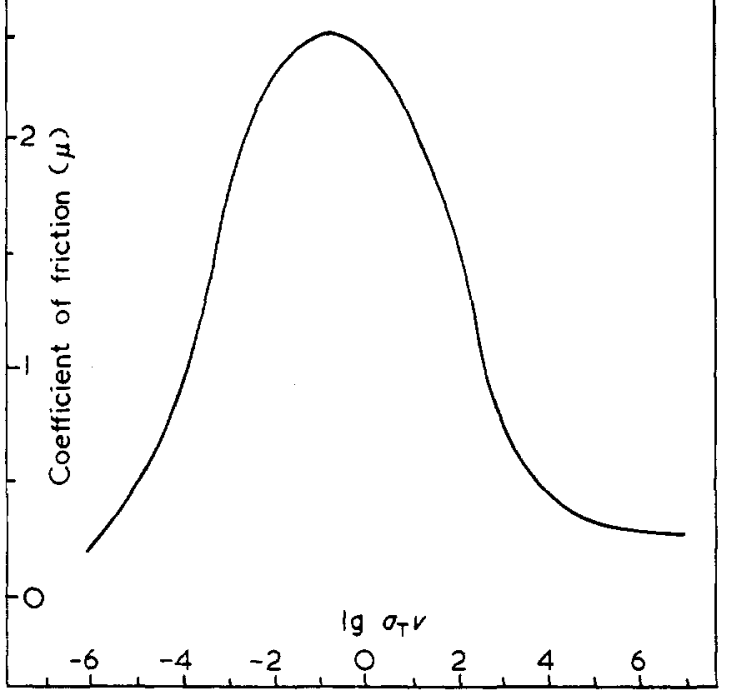


case, then $F_{\text {ad }}$ depends on bulk mechanical properties of the polymer and not on adhesion kinetics. Some authors (Bartenev and Elkin 1965 and Schallamach 1963) postulate that adhesion kinetics is responsible for either part of, or the entire, adhesive frictional behaviour. In their view, the rate of making and breaking bonds controls the magnitude of $F_{\text {ad }}$. Apparently they assume that the bonds that form also break at the original interface.

One observation at least may be explained by surface kinetics; that is the need to slide rubber a few inches before a steady state value of friction occurs. This may be due, however, to varying damping loss properties in rubber surface layers.

Whether $F_{\text {ad }}$ is controlled by adhesion kinetics or bulk mechanical properties may in the end be a moot point. There is a possibility that these two properties may be derived from the same source.

The adhesion component of friction has been shown repeatedly (Bowden and Tabor 1954, 1964) to result from strong interface bonding at the small local sites of contact between two surfaces. If bond strength is the same wherever bonds exist, the force that resists sliding is proportional to the total contact area made up of all of the minute areas of contact. A number of factors control this true area of contact. We could expect that the true area of contact of two atomically smooth surfaces would be equal to the nominal area of contact and would be very high. However, for the usual surface which is very rough on the atomic scale, contact would be limited to the highest protuberances on the surfaces. The resulting true area of contact will depend upon details of the surface profile, the magnitude of the average contact pressure and certain properties of the materials. Estimates of true contact area are available for two classic types of material behaviour. For materials that yield plastically the total true area of contact $A_{1}$ is

$$
A_{1}=\frac{K_{1} W}{3 Y}
$$

where $W$ is the load per nominal unit area, $Y$ is the yield strength of the material, and $K_{1}$ is a factor of proportionality. For contact between a sphere and an elastic material we obtain the area of contact from the Hertz contact stress equations

$$
A_{\mathrm{2}}=K_{\mathrm{2}}\left(\frac{W r}{E}\right)^{2 / 3}
$$

where $r$ is the radius of the sphere, $E$ is the (Young's) modulus of elasticity of the rubber, and $K_{2}$ is a factor of proportionality. The values of $K_{1}$ and $K_{2}$ vary with a number of conditions including surface roughness.

Rubber is usually considered to behave in an elastic manner, which is deduced from the observation that friction is proportional to average contact pressure, raised to the power of two thirds. More general conclusions on this subject can be reached after discussion of the remaining components of rubber friction.

\section{The deformation component of friction}

$F_{\text {def }}$ is a retardation force available when sliding a rubber specimen on perfectly lubricated surfaces made up of smooth bumps or protuberances (Bowden and Tabor 1954, 1964). The retardation force is due to the partial irreversibility of deformation, ie, damping loss of the rubber caused by passage of the bumps or protuberances over the rubber. This component of friction is not significant until there is sliding. The need to attribute $F_{\text {def }}$ to the damping loss of tread rubber is not often disputed. Some attempts have also been made to connect dry frictional behaviour with the damping properties of rubber, but more work is required to resolve this point.

Damping loss varies with frequency of deformation. The data are similar to the curve in figure $3 \mathrm{~b}$, except that 'damping loss' is the new ordinate and logarithmic frequency the new abscissa. It is reasonable to expect that sliding over a regular array of bumps produces a vibration in the rubber, the frequency of which would be related to sliding speed. For the topographical features of a real road, it is possible to synthesize a curve for the deformation component of friction. The large texture of a road surface is approximated by the $0.6 \mathrm{~cm}$ diameter spheres (Neville 1974). A second finer level of texture exists which has a radius of about two orders of 10 less than the coarse texture. Finally, a third very fine level of texture exists at about three or four orders of 10 less than the coarse texture. The friction performance of the three textures is shown by the solid lines in figure 4 . It may be noted that each curve in figure 4 has the same appearance as figure $3 \mathrm{~b}$, thereby suggesting that Grosch measured $F_{\text {def }}$ only. This is not the case since the data of Grosch were reproduced by this author using rubber supplied by Grosch and by doing the experiment in a vacuum of $133.3 \times 10^{-6}$ $\mathrm{N} \mathrm{m}^{-2}$. Note also that Grosch measured a coefficient of friction of 2.4 whereas $F_{\text {def }}$ seldom approaches $0.16 \mathrm{~W}$ (coefficient of friction, $\mu=0.16$ ). This point has not yet been resolved.

Figure 4 The deformation component of sliding friction for three road texture sizes over a range of sliding speed

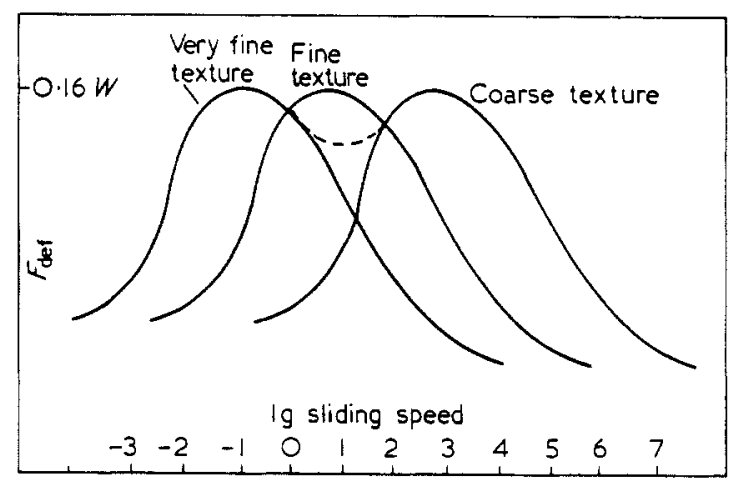

Physics in Technology January 1975 
If in the place of the discrete steps in texture size mentioned above we assume a continuous distribution, a very broad curve could be plotted without the several peaks shown in figure 4 . On the other hand, if one range of texture size is missing from the road surface, the retardation force curve could be altered as shown by the dotted line in figure 4 . This curve shows an alteration of the fine texture of figure 4 , as actually occurs on polished roads. This effect is more fully described below.

The dotted line in figure 4 may also serve as a basis for explaining the decrease in wet friction as temperature increases. The curves in figure 4 shift to the right with an increase in temperature, which has the effect of lowering $F_{\mathrm{def}}$ at moderate to slow sliding speeds. The effect is found by measurement to be the greatest on well polished roads, which also fits the curves of figure 4.

An interesting finding is that the maximum friction coefficient available from the deformation component of friction depends upon the population density of the fine protuberances on the road surface. For example, for most tyre rubbers, with a contact pressure found under most automobile tyres, $F_{\text {def }}=0.07 \mathrm{~W}$ when sliding over a tightly-packed array of spheres, while $F_{\text {def }}=0.09 \mathrm{~W}$ when sliding over the same array with alternate spheres removed. The apparent theoretical maximum $F_{\text {def }}=0.16 \mathrm{~W}$.

The deformation component of friction is difficult to separate from other components on complex surfaces. However, in spite of the low theoretical maximum value of $\mu_{\mathrm{def}}=0 \cdot 16$, this could be the primary friction mechanism at high sliding speeds on films of water. It would be expected that $F_{\text {def }}$ would be primarily influenced by the damping loss (rebound) properties of the rubber. This has been found to be true for tyres on wet roads although the effect of rubber hardness confuses the picture to some extent (Bevilaqua and Percarpio 1968).

\section{The viscous component of friction}

The viscous component of friction force, $F_{\mathrm{yis}}$ is defined to take account of the existence of a layer of either absorbed or ordinary liquid species between sliding bodies. It is presumed that this layer is thick enough to significantly reduce direct bonding, or adhesion, of the tyre rubber to the road material for example. The uniqueness of the viscous component can be demonstrated by rubbing a rubber slider on a glass that has been carefully cleaned and exposed to water vapour. A high friction force may be measured on this glass surface. In this experiment, there is no $F_{\text {def }}$ to confuse the picture because of the smoothness of glass, and no thin film of rubber remains attached to the glass.

The thickness of these absorbed water films is not known. The closest approximation can be made from the work on water absorption on lime-soda glass, a material of the same class as some road stones (Holland 1964). A surface water film, apparently $105 \AA\left(10 \times 10^{-6} \mathrm{~mm}\right)$ thick, may persist in a dry atmosphere at $23{ }^{\circ} \mathrm{C}$ and $55 \AA$ thick at $215^{\circ} \mathrm{C}$.
Very likely much thicker films would exist at lower temperatures and higher relative humidities. In addition, when two 'contacting' surfaces each have an absorbed film, the total separating film is still thicker. In practice a reduction in maximum vehicle braking force has been found on surfaces exposed to cool moist air without visible water. More careful work is needed in this area also.

Another important unknown quantity is the description of the appropriate properties of the film separating the rubbing surfaces. A thin film of lubricant may behave as a fluid of greater apparent viscosity than the bulk viscosity (Beerbower 1974). This effect is thought to be due to the nature of the bond between a liquid and solid, which is manifested by the tenacity with which liquids remain absorbed to a solid. There is some criticism of this view, but it appears to be a criticism of form rather than substance.

\section{The tearing component of friction}

$F_{\text {tear }}$ is a component of friction which takes account of the observation that some solid countersurfaces tear particles from rubber, either by cutting or by roll formation. These particles usually do not remain attached to the mating surfaces, and this mechanism can occur on contaminated surfaces where values of $\mu$ may be low. In some cases, where values of $\mu$ are high, it is easy to explain the tearing of particles from a rubber surface by high traction stress, in combination with contact stresses, which cause fracture in the rubber. Likewise, it could be argued that $F_{\text {tear }}$ is already accounted for under one of the other three components of friction. On the other hand, the deformation and thick film viscous components of friction would not ordinarily be expected to produce large wear fragments.

\section{The operative mechanisms of friction}

It is apparent from the previous sections that the adhesive mechanism of friction is not important in tyre friction. Likewise the tearing mechanism is not significant, although it is doubtless an important cause of wear. This leaves viscous drag in water films and damping loss in rubber as primary sources of tyre friction.

From the previous discussion it may be expected that the texture of the road surface is also important. In fact, the road surface texture, the tyre tread design and condition, the water depth as well as tread rubber properties, are all vital quantities in the control of skid resistance. The following scenario is the basis for the analysis currently being done by several workers.

A rolling tyre can be viewed as a device that places tread elements on the top of a water film, applies a load for a period of time and then reverses the process. Throughout the contact excursion the fluid film thins and there is an increasing viscous drag force potential, which is not realized until there is slip. When slip occurs there is some film thickening probably because of turbulence at the 
inlet (or front) region of the tyre. Figure 5 illustrates this. It shows a view up through a glass plate on which a tyre is rolling at approximately $17.88 \mathrm{~m} \mathrm{~s}^{-1}$. Any point on the tyre is moving upwards, and the area showing the tread pattern clearly is the load bearing, thin water film region. More of the tyre appears to be behind the centre of contact, than ahead. This is because the water is obscuring the view. (This may be part of the explanation for the negative slope of the curves in figure 2.)

The average water film thickness under the tyre will obviously be greater when the water ahead of the tyre is deeper, and when less time is available to expel water from under the tyre as for example when vehicle speed is high. On the other hand, the average water film will be thin when contact pressure is high and where water can be more completely expelled from the tyre-road interface. This may be accomplished by a long and narrow contact region and/or by the existence of grooves or pathways on the tyre or road surface (or both) to aid the flow of water.

A complete analysis of the water expulsion rate should therefore take all of the above factors into account. This has proved to be very difficult. To date the best analytical work in this area is due to Browne. In essence his work consists of a hydrodynamic analysis for two mathematically smooth surfaces. A major problem in the analysis has been to express the entrance shape properly. However, he has arrived at a computer solution for average water film under a tyre which has been verified by experiment. The magnitude of this accomplishment should not be overlooked. Yeager (1974) has verified this experimentally by photographing the contact event where a full scale automobile tyre is run over a glass plate

Figure 5 A view of the tyre-road interface in the wet (Dunlop (UK) Ltd.)

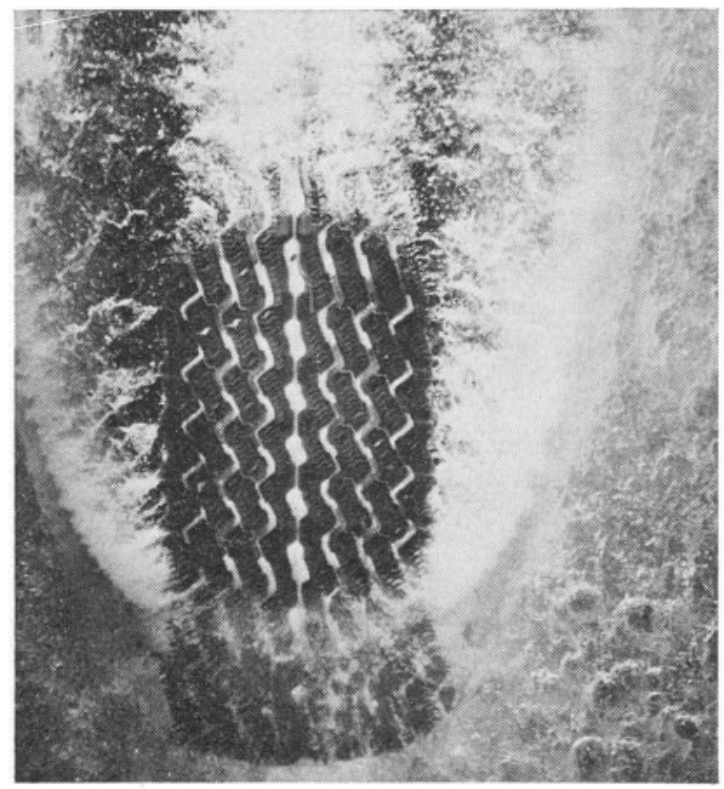

up to relatively high speeds.

By experiment Yeager has shown the effect of tyre tread pattern, tyre loading, tyre shape (with speed, inflation, and so on), but some of these quantities are difficult to express mathematically. Browne and Yeager have not yet been able to include the effect of road surface texture in their work. This step is very useful because it is the road surface texture that interacts with tyre rubber to provide the damping loss component of friction. In all likelihood the damping loss component is the major component when friction is low. However, the work of Browne and Yeager is a milestone in the literature.

Another analysis, to be discussed below shows the role of road surface texture details in isolation from the behaviour of tyre as a whole. The latter analysis includes the effect of tyre rubber properties, however. It is an elastohydrodynamic analysis for water film thickness between a single rigid sphere, and rubber which is a viscoelastic substance. The analysis is therefore referred to as a viscoelastohydrodynamic analysis.

The analysis begins with the observation that road surfaces, though of complex texture, are made up of protuberances or asperities with spherical tops. These asperities have various radii and heights, and they are distributed on the road surface in various arrays. The analysis is done on the basis of each spherical asperity expelling the water in its pathway. Obviously if an asperity is surrounded by others which are also expelling water in its pathway a point is reached when a limitation of expulsion of water is reached, but this case has not been dealt with. In the extreme, the tyre will behave like the mathematically smooth surface used by Browne and Yeager.

The viscoelastohydrodynamic analysis is developed completely in a recent paper (Gujrati and Ludema 1974), and will not be reproduced here. The equation consists of two parts, and it is assumed that there is no significant cross-coupling between terms. Thus the sum, $\mu_{\mathrm{def}}+\mu_{\mathrm{vis}}=\mu_{\mathrm{tot}}$, which is the coefficient of friction for a sphere sliding on rubber immersed in water. The two calculated components are shown plotted against speed in figure 6 , for a polybutadiene rubber at $25^{\circ} \mathrm{C}$. Experimental values for each component are also shown in figure 6. The data for $\mu_{\mathrm{def}}$ are obtained by measuring the rolling friction of a steel sphere on rubber with a particular load applied. The data for $\mu_{\text {vis }}$ are obtained by sliding the hemispherical end of a rubber cylinder against a glass plate under water, with the same load. In figure 6 it can be seen that there is a better correlation between $\mu_{\mathrm{def}}$ and $\mu_{\text {rolling }}$ than for the viscous situation. The discrepancy doubtless lies in an erroneous assumption concerning wettability as explained below. There are no generally acceptable equations for a viscoelastic strain or stress field equivalent to the Hertzian equations for elastic materials.

Figure 6 also shows a curve for the total friction of a steel sphere sliding on rubber submerged in 
water. It should be noted in particular that curve $\mathrm{E}$ departs considerably from the sum of both calculated and measured components at low speeds. In fact, the maximum friction found at low speeds was found to be the same for the case of no visible wetness. It appeared that water might be expelled from the points of intimate contact but by a mechanism other than accounted for in hydrodynamic calculations. One force suspected was surface forces or contact angle forces. Thus a series of fluids was prepared which contained surface active agents which aided wetting to rubber. This had the effect of reducing the speed at which experimental friction values deviated from theoretical values. When isopropanol was used as the 'lubricant' in the place of water there was no inflection in the curve. Thus it appears that the assumption of perfect wettability in equations of hydrodynamics is not always valid.

\section{Future developments}

Neither the analysis for the events around a single asperity, nor the analysis for the water expulsion from under a smooth tyre are sufficient for use in the development of an ideal tyre-road pair. The viscoelastohydrodynamic analysis is useful only where water films are very thin and freely expelled, whereas the equations for water expulsion do not take account of road texture or rubber properties.

Fortunately for the public, tyres and roads have been improving without benefit of analysis. Doubtless skid resistance technology could proceed toward an optimum more quickly if the tyre and road industries were to work together more often. Each industry has its own battery of tests, and only rarely are they exactly the same for each. It is quite evident that the tyre industry and the road building industry are strongly influenced by considerably different technical disciplines.

The influence of rubber properties is complex. However, in summary it can be said that the choice of base polymer and of the ingredients used in compounding can affect frictional properties not only through their influence on such physical characteristics as hardness, damping loss and surface roughness, but also by changes in chemical adhesion and changes in wettability by water. In view of the several constraints on the practical range of tyre rubber properties the available range of friction is not large. By far the most important variables are the nature of the road surface texture and the tread pattern. Again, there are limits to tread pattern design defined by noise generation, uneven wear, chunking out and so on. There are limits on road surface texture as well and these relate to noise generation and tyre wear. However, it appears that in the United States at least, road building practices are more in need of improvement than is tyre design. At this late date in history some road builders still take pride in producing a beautiful (smooth) road surface. One can be complimentary of such a view until the first event requiring high skid resistance!

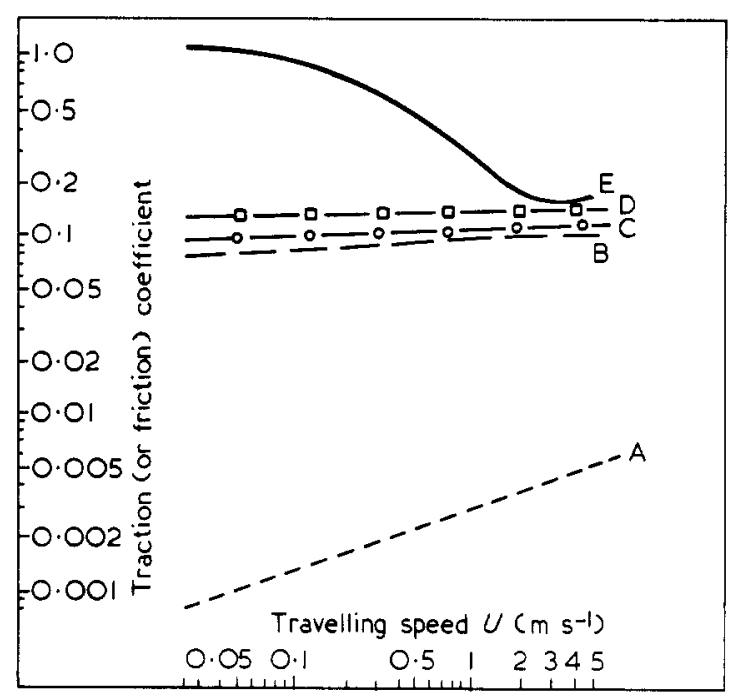

Figure 6 The coefficient of friction of spheres on wet rubber over a range of sliding speed using: A theoretical viscous component (assuming perfect wetting of rubber). $B$, theoretical deformation component; $C$, measured viscous component (using isopropanol as the lubricant) with a rubber sphere on a glass plate; $D$, measured deformation component (by rolling a steel ball on a rubber slab); E, total measured friction (by sliding a steel ball on a rubber slab immersed in water

\section{References}

Bartenev G and Elkin A 1965 Wear 8 p8

Beerbower A 1974 Interfacial Phenomena in Petroleum Recovery Am. Inst. Chem. Engrs. Tulsa Oklahoma paper 400

Bevilaqua E and Percarpio E 1968 Rubber Chem. and Tech. 41832

Bowden F P and Tabor D 1954 Friction and Lubrication of Solids 1 (Oxford: University Press)

Bowden F P and Tabor D 1964 Friction and Lubrication of Solids 2 (Oxford: University Press)

Browne A L $A$ mathematical analysis for pneumatic tyre hydroplaning 5 th annual Kummer lecture to Commitee E-17 of Am. Soc. Test. Mat. and approved for publication by ASTM 1916 Race Phila $\mathrm{Pa}$

Byrdsong T A 1968 NASA Tech Note D-4602 p 44

Grosch K A 1963 Proc. R. Soc. A 274 p21

Gujrati B D and Ludema K C 1974 The Physics of Tyre Traction ed. D F Hays and A A Browne (New York: Plenum Press) p 197

Holland L 1964 The Properties of Glass Surfaces (London: Chapman and Hall) p 231

Holmes K E and Stone R D 1968-9 Proc. Inst. Mech. Engrs (London) 183 pt $3 \mathrm{H}$

Ludema K C and Gujrati B D 1973 Analysis of the Literature on Tyre Road Skid Resistance American Society for Testing and Materials STP No. 514

Neville G 1974 Transport and Road Research Loboratory Report $621 \mathrm{p} 4$

Schallamach A 1963 Wear 6375

Yeager R W, Hays D F and Browne A A 1974 The Physics of Tyre Traction ed. D F Hays and A A Browne (New York: Plenum Press) p 25 